\title{
,Schon schnellten sich von Oskars Zunge einige französische Wörtchen': Die Blechtrommel in Frankreich
}

\author{
Stéphanie Vanasten
}

\begin{abstract}
In general, the French-speaking critique did not lack in superlatives when discussing the overwhelming breakthrough and the continuously respected presence of Grass's first novel Die Blechtrommel, which was translated in 1961 as Le Tambour. The book sets a standard for the opinions expressed in later reviews and comments. While research on the journalistic reception of Grass's later novels in France reveals a differential appreciation in comparison with his earlier work, the French-language criticism and readership continued to support Grass's work. Although there exists a considerable number of studies on Grass's critical reception and on his international significance, the French reception of Le Tambour remains in this respect surprisingly ill-documented and underinterrogated. The aim of this article is, first of all, to provide a critical overview of the successful cultural transfer that Le Tambour represents in France and to gain insight into the possible reasons for this in view of a transnational reception perspective. Secondly, the present article examines one particular and unexplored segment of the work's critical reception: the memory and canonization of Le Tambour throughout the national teaching certification programme of the Agrégation de lettres modernes in France. Our research into this subject shows how Grass's novel, within the frame of a picaresque reading, does not so much follow the journalistic reception, but - according to our hypothesis - appears to emanate, already from the very beginning, an shared sense of aesthetics that formed for the French target public a European entry into the work, detached from the solely German matter.
\end{abstract}

In Frankreich genießt der junge Schriftsteller Grass vor dem Erscheinen seines inzwischen weltberühmten Romans Die Blechtrommel keine Bekanntheit. „Ich schrieb für kein Publikum, denn ein Publikum kannte ich nicht", blickt der Schriftsteller 1973 auf die Pariser Entstehungszeit (1956-196o) der Blechtrommel

(C) STÉPHANIE VANASTEN, 2016 | DOI 10.1163/9789004291898_010 
zurück$^{1}$. Sein Magnum Opus eröffnete, wie man weiß, den Weg zum Erfolg und bereitete eine Flut von späteren Kommentaren vor.

Den Blick auf Frankreich richtend ist es in der Kritik ${ }^{2}$ inzwischen fast zum Gemeingut geworden zu bemerken, dass der Roman auf Danzig und „die verlorene Provinz" bezogen ist ${ }^{3}$, wohl aber in Paris entstanden ist und auch noch beiläufig nach Frankreich als Schauplatz der Handlung führt: Solch eine diskursive Aneignung macht einen Anteil an der Auseinandersetzung mit der fremden Kultur aus. War das Buch aber hauptsächlich in einem spezifischen nationalen bzw. regionalen Kontext verwurzelt, erreichte es trotzdem - zu Grass' eigener Überraschung - ein übernationales Publikum und „verflüchtigte sich“, um es mit dem Autor zu sagen, schnell international. ${ }^{4}$

Als Buch im Nachkriegsdeutschland zunächst wenige, dann zum Teil heftige Reaktionen sogar Skandale erregend, wurde Die Blechtrommel besonders im Ausland, allen voran in Frankreich, als Meisterwerk gefeiert. ${ }^{5}$ „Im Ausland geschätzt, im Inland gehasst“, karikierte Grass selbst die Lage in einem berühmten und vielzitierten Interview aus dem Jahre 1977. Scheinbar folgte die französische Literaturkritik in ihrem Urteil nicht der deutschen, sondern ging anders als diese mit dem großen Erzählwerk um. Unmittelbare Rezensionen waren selten negativ. Kaum ein Jahr nach dem Erscheinen in französischer Übersetzung wurde der Roman 1962 bereits mit dem renommierten Prix Medicis Etranger ausgezeichnet. „Den Status des Klassikers hat er [Grass] mit seinem Erstlingsroman bereits erreicht“, blickt Corina Ortuño Stühring auf die Blechtrommel zurück, als sie die journalistische Rezeption des späteren Romanwerks in Frankreich und Deutschland untersucht. ${ }^{6}$ Bereits 1975 stellte der Schriftsteller Michel Tournier, Mitglied der Académie Goncourt, in Le Monde fest: „Il [Le Tambour] connut un succès considérable en France. Le recul de quinze années permet aujourd'hui une lecture mieux pondérée. Le doute n'est pas permis: l'oeuvre majeure de Günter Grass reste l'une des plus significatives de toute la production allemande d'après-guerre. ${ }^{\text {7 }}$ Bis in neueren Publikationen gilt der Schriftsteller in Frankreich als einer der besten und meistübersetzten

\footnotetext{
1 Grass (1997) 328.

2 Vgl. Serrier (2006), George (2008), Mannoni (2000).

3 Grass (1997) 323 .

4 Grass (1997) 323.

5 In seiner Besprechung des Romans vom 27. Dezember 1961 in Le Monde drückte Pierre-Henri Simon es treffend aus : „[...] la forte impression, nuancée de scandale en Allemagne où le roman de M. Grass a paru il y a deux ans, et aujourd'hui produite par sa traduction en France $[\ldots]^{\prime \prime}$.

6 Ortuño Stühring (2013) 295 .

7 Tournier (1975).
} 
deutschsprachigen Autoren der Nachkriegszeit ${ }^{8}$, und sein großes Jugendwerk als „un des romans les plus lus du siècle“.9 Auch im heutigen französischen Literaturbetrieb ist Grass „seit langem ein Begriff“, der den Lesern nicht mehr vorgestellt werden muss. ${ }^{10}$

In dieser Hinsicht kann der Fall Blechtrommel mit Gewinn als Fremdkulturvermittlung oder als Beispiel für erfolgreichen ,kulturellen Transfer' rezipiert werden, wie ihn Michel Espagne bestimmt: „[le] passage d'un objet culturel d'un contexte dans un autre [avec] pour conséquence une transformation de son sens, une dynamique de resémantisation, qu'on ne peut pleinement reconnaître qu'en tenant compte des vecteurs historiques du passage ". ${ }^{11}$ Die früheErfolgsgeschichte der Blechtrommel, auch wenn sie sich wie ein Ölfleck auf die weitere Rezeption verbreitet, steht jedoch einer um die Jahrhundertwende nicht durchweg positiv über Grass urteilenden französischen journalistischen Literaturkritik entgegen, wie Corina Ortuño Stühring im Rahmen ihrer Dissertation aus ihren Untersuchungen zu dem Zeitraum 1995-2006 schlussfolgerte. ${ }^{12}$ Eine mögliche Begründung dafür sieht die Literaturwissenschaftlerin nicht in einer geringen qualitativen Bewertung der durch die Rezensenten besprochenen Werke, sondern allgemeiner in den Grenzen der Aneignung fremden Kulturgutes: Wenn das Verstehen der (im vorliegenden Fall mit Geschichte überladenen) Romane aus dem späteren Werk dem französischen Leser bzw. Rezensenten schwerfalle, so nicht unmöglich werde, bleibe der Prozess der Aneignung der Fremdkultur aus. Auch Marion George bezeichnet übrigens „weitausgehende historische Konstruktionen als Hindernisse für die Lesbarkeit“. ${ }^{13}$ So könne ein schwer transferierbares Werk zu einer negativen Wertung oder zu einer archaisierenden Wahrnehmung führen, „bei der keine oder nur geringfügige Anpassungsprozesse bereits vorhandener Fremdbilder an die Gegenwart stattfinden". ${ }^{14}$

Der Verweis auf die Gesetzmäßigkeit solcher systemisch-theoretischen Standpunkte kann den Erfolg der Blechtrommel in Frankreich allerdings nur dann erklären, wenn man davon ausgeht, dass der Roman damals einen besonderen Anschluss an Frankreichs literarisches Feld und seine Wertungsmechanismen gefunden haben muss. Ist Die Blechtrommel aber nicht auch wie

$8 \quad$ George (2008) 304.

9 Mannoni (2000) 132. Aktuelle Auflagenzahlen und Lesestatistiken waren für Frankreich beim Herausgeber leider nicht erhältlich.

$10 \quad$ Ortuño Stühring (2013) 257.

11 Espagne (2013).

12 Ortuño Stühring (2013) 251, 260.

13 George (2008) 309.

14 Ortuño Stühring (2013) 263. 
das späte Grass'sche Werk, wie auch Ortuño Stühring weiter ausführt, weitgehend von der Geschichte und der Vergangenheitsbewältigung geprägt? Laut Thomas Serrierbeweist eben die gute Aufnahme von ImKrebsgang in Frankreich (En crabe, 2002), dass das Verständnis der historischen Fakten kein Hindernis darstelle und stattdessen das zunehmende Bewusstsein der Franzosen für Grass' eigenen Umgang mit komplexen und schwierigen geschichtlichen Themen, der dem französischen Lesepublikum lange fremd war, beweise. ${ }^{15}$ Wie lässt sich dann, rückblickend auf die differenzierte Appreciation, die positive Wertung des ersten großen Romans der Danziger Trilogie erklären, übrigens auf lange Sicht? Denn es gilt ohnehin, Die Blechtrommel stelle „die wichtigste Referenz [...] in der französischen Rezeption des Grass'schen Werkes [...]“ dar ${ }^{16}$, „de[n] bekannteste[n] aller Grass-Romane [...] wie schon mehrmals anhand von französischen Feuilletons aufgezeigt werden konnte“. ${ }^{17}$

Ein Grund könnte darin liegen, dass die französische Literaturkritik von einer anderen Ästhetik geprägt sein könnte, die sie ,besser' bzw. anders in die Lage versetzt, die von der Barock- und Rabelais-Sprache geprägte Poetik der Blechtrommel - auf welche die französische Kritik auch im Laufe der Jahre explizit eingeht - zu erfassen (ohne hier von der möglichen Nähe zu einem anderen, in Frankreich genauso zur Doxa gehörenden Text zu sprechen, auf den Grass auch selber hinwies, nämlich den Ulenspiegel von Charles de Coster, den Grass nach eigener Aussage noch vor Rabelais gelesen hat ${ }^{18}$, oder von der Tradition des Schelmenromans oder der Romantik, der manche Kritiker die ersten Grass-Romane zuordnen). ${ }^{19}$ Gingen die Franzosen als Interpreten somit (anders als die Deutschen?) unproblematischer, d.h. mit einer Öffnung der Perspektive, an die ästhetischen Aspekte des Textes und seine Provokation heran? Und wäre Grass' Pariser Aufenthalt vor dem Hintergrund seiner Entdeckung von Rabelais über Paul Celan in den 5oer Jahren für die Nähe zum französischen Publikum entscheidender gewesen, als die damalige intellektuelle Einsamkeit, die er in Interviews angibt, vermuten lässt?

Vor diesem Hintergrund erweist sich die Vermittlung der Blechtrommel in Frankreich in Bezug auf die Problematik des kulturellen Transfers und transnationaler Rezeptionsforschung als höchst aufschlussreich für Untersuchungen. Nach Erklärungen für die Art der Resemantisierung durch Rekontextualisierung und eventuelle Kanonisierung in Frankreich wird im

\footnotetext{
$15 \quad$ Serrier (2006) 85.

16 George (2008) 304; vgl. auch Serrier (2006) 82.

17 Ortuño Stühring (2013) 265.

18 Grass (1999) 63.

19 Casanova (2002), zit. nach George (2008) 309.
} 
Folgenden an den historischen Schnittstellen der Literaturkritik gesucht, und zwar in einem besonderen Segment der Rezeption, das in den Kontext der in Frankreich anspruchsvollen Lehramtprüfung (concours oder staatliche Aufnahmeprüfung), Agrégation genannt, eingebettet ist.

$\mathrm{Zu}$ einem Gesamtbild der französischen Rezeption ließen sich die zu den untersuchten literaturkritischen Kanälen erlangten Einsichten allerdings nur verdichten, wenn auch das Lesepublikum mit einbezogen werden kann. Die Rolle der Literaturkritik kann heutzutage nicht mehr ausschließlich am Urteil der klassischen Printmedien-Rezensenten gemessen werden. ${ }^{20}$ In seiner 1999 erschienenen Grass-Monografie hob der französische literarische Journalist und Übersetzer Olivier Mannoni zudem hervor, wie oft Grass von der Literaturkritik angegriffen worden sei, jedoch immer wieder - den Absatz von Grass' Büchern als Beweis anführend - von seinen Lesern unterstützt und weltweit von Verlagen angekauft und übersetzt wurde. ${ }^{21}$ Auch Marion George zufolge hat sich die französische Öffentlichkeit, als eine Kontroverse um Grass' politische Meinungen entstand, „fast immer an seine Seite gestellt“. ${ }^{22}$ Eine empirische Leserforschung in Bezug auf das französischsprachige Feld - insbesondere bei den Agrégés und den von ihnen ausgebildeten (Leser-) Generationen - würde den Rahmen dieses Beitrags sprengen, ist aber daher insbesondere im Hinblick auf Frankreich - als Desiderat für zukünftige Forschung zu benennen.

\section{Grass international? Le Tambour als verborgene Tiefe des Eisbergs}

Eine Menge von literaturwissenschaftlichen Studien hat den Durchbruch und das weitere Auftreten Grass' und seiner Prosa in den deutschen und internationalen Feuilletons verfolgt. Die Sekundärliteratur über den Nobelpreisträger ist heutzutage kaum noch zu verzeichnen und statistisch beeindruckend. „Rezeptionsgeschichten zu Grass-Werken gibt es wie Sand am Meer“, meinte ein Rezensent. ${ }^{23} \mathrm{Zu}$ der französischen Blechtrommel-Rezeption und deren entscheidendem Nachwirken für Grass' späteres Werk in Frankreich, vielleicht sogar über seine Grenzen hinaus, und für eine mögliche Rückwirkung auf die deutsche bzw. internationale Rezeption (was Espagne ,tiers impliqués' nennt) hat sich die Literaturwissenschaft erstaunlicherweise kaum geäußert. Zwar

\footnotetext{
$20 \quad$ Joosten (2012) 10.

21 Mannoni (2000) 522, 175.

$22 \quad$ George (2009) 310.

23 Frizen (2006) 96.
} 
werden zur Besprechung von Grass' ausländischem Ruf gelegentlich Äußerungen bezüglich Frankreich herangezogen, um das internationale Grass-Bild zu bekräftigen, kaum richtet sich aber dabei der Blick auf die Spezifizität der französischen Grass- bzw. Blechtrommel-Rezeption. ${ }^{24}$ In dieser Hinsicht besteht angesichts Grass' ,international standing ${ }^{25}$ und des allgemein expandierten Angebots an literaturwissenschaftlichen Grass-Beiträgen zwischen dem besonderen und fast historischen französischen Interesse an Grass, dem außergewöhnlichen Umfang des Werkes in französischer Übersetzung und Grass' bekannter Orientierung, sicherlich in den frühen Jahrzehnten, an Frankreich einerseits und der geringen literaturwissenschaftlichen Nachfrage bezüglich der (frühen) positiven Rezeption und der Wirkung des Grass'schen Werkes in Frankreich andererseits eine erstaunliche Diskrepanz.

Somit bestätigt sich für Frankreich, was schon Rebecca Braun und Frank Brunssen zu ihrem Sammelband Changing the Nation. Günter Grass in international Perspective bewegte: Der Schriftsteller gilt als „one of the few international figures of our times. [...] This very internationalism, however, has attracted surprisingly little sustained analysis from Grass scholars". ${ }^{26}$ Demgegenüber muss gesagt werden, dass bei allen Unterschieden zwischen den Rezeptionsländern gerade in Frankreich der Roman Ein weites Feld (1995; Toute une histoire, 1997) proportional außergewöhnliche Aufmerksamkeit auf sich gezogen hat. In Changing the Nation wurde der spezielle Themenkomplex ,Grass und Frankreich' relativ wenig beachtet, mit Ausnahme von Julian Preece, der in seinem komparatistischen Beitrag eben die vernetzten französisch-deutschen Beziehungen in Ein weites Feld erforschte und aufzeigte, wie der Roman für einen transkulturellen Dialog nicht nur zwischen Individuen, sondern auch zwischen Nationen Modell steht. ${ }^{27}$ Erkenntnisse im Sinne einer (vergleichenden) Rezeptionsgeschichte von Grass' Werk blieben in dem Rest des Bandes vorwiegend auf die englischsprachigen Länder beschränkt. ${ }^{28}$

24 So z.B. Mews (2008).

25 Braun/Brunssen (2008) 10.

26 Braun /Brunssen (2008) 9.

27 Braun/Brunssen (2008) 16. Interessant ist, dass gerade die französische Zeitung Le Monde in ihrer positiven Besprechung des Romans bedauerte, Grass habe nicht genug zu einem erneuten Dialog zwischen Ost und West angeregt und dadurch vermutlich die heftige Reaktion auf sein Werk in Deutschland ausgelöst. (Pierre Deshusses (1997), zit. nach Ortuño Stühring (2013 ) 255). Diese Differenzierung illustriert u. a., wie unterschiedlich die Wertung zwischen literaturkritischem und akademischem Urteil erfolgen kann.

28

Die USA in dem Aufsatz von Siegfried Mews, Australien und Neuseeland in dem von Alexandra Ludewig. 
Somit spiegelte sich hier ein Grass-Rezeptionsmuster wider, das Siegfrid Mews schon früher, jedoch eher beiläufig, im Hinblick auf Frankreich beobachtet hatte. In Günter Grass and His Critics (2008) stellte Mews ausgehend von der Untersuchung von Henrik D.K. Engel (Die Prosa von Günter Grass in Beziehung zur englischsprachigen Rezeption, 1997) fest, dass die Sekundärliteratur zu Grass im Französischen quantitativ weit hinter den deutschsprachigen Ländern, den USA, Kanada und Großbritannien liege. Sich auf Engel stützend, erwähnte Mews für Frankreich zehn zwischen 1960 und 1989/1990 erschienene Monografien und Dissertationen zu Grass. ${ }^{29}$ Diese quantitative Berechnung ist zeitlich umso mehr ergänzungsbedürftig, als dass - neben dem besonderen Interesse in Frankreich für den deutsch-französischen Wenderoman Ein weites Feld - seither ein besonderes Phänomen der literaturkritischen Überlieferung und Wirkung in Frankreich neue Grass-Publikationen auf den Markt gebracht hat: die Aufnahme von zwei Grass-Romanen als verpflichtende Lektüre in die renommierten nationalen Prüfungsprogramme CAPES (Deutsch, 2002 für Ein weites Feld) und Agrégation de Lettres modernes (vergleichende Literatur, 2004 für Le Tambour). Im Falle von Ein weites Feld (Toute une histoire, 1997) hat dies zu nicht weniger als drei Bänden in einer Zeitspanne von einem Jahr geführt $^{30}$, im Falle der Blechtrommel - interessanterweise nachträglich, mit möglicher Rückwirkung von Ein weites Feld oder des Nobelpreises, also von zwei Momenten, die nach 1995 in der französischen Presse dominieren ${ }^{31}-\mathrm{zu}$ einer komparatistischen (Teil-)Monografie und wenigstens einem Aufsatz. ${ }^{32}$ Trotzdem ist Mews darin zuzustimmen, dass die meisten Untersuchungen innerhalb der Grass-Forschung (zumindest in europäischen Sprachen) auf Grass' coverage und Rezeption in deutschsprachigen bzw. englischsprachigen Medien fokussieren. ${ }^{33}$

\footnotetext{
29 Mews (2008) 10-11.

30 Wellnitz (2001); Lartillot (2001); Quéval (2001).

$31 \quad$ George (2008) 305 .

32 Pinçonnat e.a. (2003); Ballestra-Puech (2004).

33 Gegen Mews' Annahme, dass „scholarly contributions written in German, English or French most likely constitute the overwhelming majority of the worldwide critical appraisals devoted to Grass's writings“ (S. 7), erhebt sich aus der Untersuchung von Thomas Serrier der Einwand, Polen müsste auch mit zur Reihe gehören: „Günter Grass est traduit dans plus de vingt langues. La France ne se distingue sans doute pas de la moyenne des autres pays de réception, comme les Etats-Unis ou la Grande Bretagne. Les deux terreaux les plus réceptifs et les plus sensibles sont indéniablement l'Allemagne et, une nouvelle fois, la Pologne: c'est à Varsovie, et non pas à Paris, que Grass jouit du statut de véritable icône de la réconciliation entre les peuples." Serrier (2006) 82.
} 
In seiner Monografie erwähnt Mews selbst einige Meilensteine der akademischen bzw. essayistischen französischen Grass-Kritik ${ }^{34}$, Pressereaktionen gehören jedoch nicht zum Hauptfokus seiner Untersuchung, die er allgemein als "considerably less inclusive to France“ präsentiert. ${ }^{35}$ Ähnliches gilt für die Studie von Wilhelm Johannes Schwarz über den Erzähler Günter Grass (1969), dessen „Übersicht über die Grass-Kritik“ zuerst einige Quellen auf Französisch aufzählt, doch keine aus der französischen journalistischen Kritik erwähnt. Auf einen mathematischen Vervollständigungsversuch im Hinblick auf eine wissenschaftliche Bibliografie der französischen Grass-Kritik wird hier verzichtet, da nicht alle verfügbare Quellen zielgerichtetes Material zur journalistischen Rezeption der Blechtrommel anbieten bzw. auf ein in einem anderen Rezeptionsland vergleichbares Phänomen im Kontext des Hochschulunterrichts wie die auf die Agregation hinzielende (akademische) Literaturessayistik und Rezeption eingehen. Hinsichtlich der Herangehensweise wird folglich bevorzugt, was auch Mews sinnvoll erschien: „To be sure, a pure quantitative analysis has to be supplemented by an exploration of the reasons why a specific author or work succeeds or fails in a foreign linguistic, cultural and political environment. ${ }^{\text {“36 }}$

Mit seinem 1999 erschienenen Buch Günter Grass. L'honneur d'un homme legte der französische literarische Journalist und Übersetzter Olivier Mannoni einen ersten bedeutenden Versuch vor, die Reaktionen der deutschen Massenmedien auf Grass' Werk - unter Berücksichtigung der für französische Begriffe noch nie dagewesenen Mediendebatte um Ein weites Feld - in einem vergleichenden-differenzierenden Bezug auf Frankreich in seine Arbeit aufzunehmen und für ein breites französisches Publikum darzulegen. Fokussiert Mannoni in seinen chronologischen Ausführungen nicht besonders auf die Rezeption von Die Blechtrommel, so erfährt man hier viel zum Hintergrund des diachronen Grass-Image jenseits des Rheins. Der Verfasser verfolgt aber keine wissenschaftlichen Intentionen, und es ist zu fragen, ob die durchweg positive und dauerhafte Wertung, die Mannonis Monografie von Grass' Werk in Frankreich aufweist, womöglich von dem persönlich-bewegten Ton des Verfassers (nicht zuletzt als Liebhaber der deutschen Sprache und Befürworter von Grass) gefärbt worden ist und allzu sehr dramatisiert wurde. ${ }^{37}$ Bemerkenswert ist, dass auch Marion George ,den Grundton' der Kritik

\footnotetext{
34 Plard (1980) \& (1984); Leroy (1969) \& (1970-71); Mannoni (2000).

35 Mews (2008) 7 .

$36 \quad$ Mews (2008) 11 .

37 Guissard (2000) 16; Serrier (2006) 84.
} 
in Frankreich als ,durchgehend positiv bewertet. $^{38}$ Sie erwähnt zudem einen interessanten Parameter, auf den Mews und Braun schon früher hingewiesen haben, und zwar darauf, dass der Autor auch selber dazu beigetragen habe. So soll das lange Metropolis Spezial-Interview von Grass, das mehrmals auf ARTE ausgestrahlt wurde, für die Grass-Perzeption der Franzosen entscheidend gewesen sein. ${ }^{39}$ George schließt ihren Beitrag aber mit einem Aufruf zur Vorsicht, und mahnt, dass es der Rezeptionsgeschichte überlassen werden soll, über das Grass-Bild das letzte Wort zu sprechen, denn „die zeitliche Nähe verführt zur Parteinahme und verstellt gelegentlich den Blick auf das Wesentliche". 40

So nuanciert die 2013 erschienene Dissertation von Corina Ortuño Stühring anhand empirischer Quellenauswertung, wie schon vorher angedeutet, ein über die Zeiten hinweg durchweg positives Bild von Grass' Werk in Frankreich. Insbesondere nahm die Studie, wie ihr Titel Die Kritik der Gegenwart. Eine systematische Analyse deutsch-französischer Literaturkritik am Beispiel von Michel Houellebecq und Günter Grass verspricht, die Rezeption von Grass' Spätwerk, und zwar des in literaturkritischer Hinsicht besonders intensiven Segments von Ein weites Feld (1995) bis zu Im Krebsgang (2002), in Frankreich und Deutschland ,gegenüberstellend-vergleichend ' in den Blick. ${ }^{41}$ Im Vergleich zu Deutschland gewinnt Ortuño Stühring aus ihrer Untersuchung ähnliche Einsichten wie Mannoni: Die französische Kritik habe Grass auch in Bezug auf sein Spätwerk bedeutend mehr gefeiert als die deutsche. Reagierte sie manchmal lau auf die Publikation bestimmter Romane, war der Empfang bzw. die Wertung immerhin positiver als bei den deutschen Kritikern. ${ }^{42}$ Ortuño Stühring folgert dennoch nuanciert aus ihrer Rezensionsanalyse, dass dabei „[...] nur von Tendenzen gesprochen werden kann, da sie [die französischen Kritiker] sowohl in den Kritiken zu Ein weites Feld als auch zu Im Krebsgang größtenteils in der Neutralität verharren und nur ab und zu ein Lob durchschimmern lassen. In der Tendenz zur Positivbewertung sind sich die französischen Kritiker jedoch mit den deutschen größtenteils einig [...]“ “43

Auch wenn die frühe Romanrezeption nicht zu ihrem Fokus gehört, erweist sich aus Ortuño Stührings Studie, wie Die Blechtrommel als aus vergangener Zeit stammender Maßstab der positiven Wertung vor dem Hintergrund späte-

\footnotetext{
$38 \quad$ George (2008) 310.

39 George (2008) 301.

$40 \quad$ George (2008) 310.

41 Erstaunlicherweise allerdings ohne dabei auf Mannoni zu verweisen.

42 Mannoni (2000) 374, 398, 504 ; vgl. Ortuño Stühring (2013) 260 ff.

43 Ortuño Stühring (2013) 260.
} 
rer Rezensionen weiterhin eine Rolle spielt und somit das späte AutorenImage von Grass in der französischen Presse lenkt. Denn Ortuño Stühring gibt an, dass die französischen Literaturkritiker - anders als die deutschen - zwischen Grass' schriftstellerischer Arbeit und dem Werk, das sie besprechen, differenzieren, wobei sie „durch die ausdrücklich positive Hervorhebung älterer Romane [...] an tradierte Perzeptionsmuster anknüpfen“. ${ }^{44}$ Was diese ,tradierten Perzeptionsmuster' sind, ist über allgemeine Wahrnehmungen hinaus anhand einer empirischen Quellenauswertung im französischen Kontext eben zu hinterfragen, umso mehr, als sich die frühen Rezeptionsmuster nachträglich, infolge einer Form von Beharrung oder Änderungswiderstand, hartnäckiger erweisen als gedacht.

Nicht zuletzt haben Thomas Serrier 2006 und Marion George 2008 in zwei kürzeren, doch zielgerichteten Auseinandersetzungen mit dem Thema,Günter Grass und Frankreich' das französische Bild des Grass'schen Werks im Laufe der Zeit erforscht. Geschieht das zwar nicht unter besonderer Berücksichtigung der Blechtrommel, unternehmen beide dennoch einen interessanten Versuch, Leseinteressen oder literaturkritische Umgangsmuster mit Grass und seinem Werk generell für Frankreich zu skizzieren. Dabei verweisen sie in erster Linie auf politische Lektüren, mitunter auf eine geschärfte Aufmerksamkeit für den komplexen Prozess der Vergangenheitsbewältigung, der historischen Konstruktionen und des Gedächtnisprozesses. Solche Lektüren weisen aber, so George, das literarische Urteil als zweitrangig zurück. ${ }^{45}$ Daneben halten sowohl Serrier als auch George, ohne jedoch aufeinander zu verweisen, bei den französischen Kritikern bzw. Lesern eine Faszination für ästhetische Präokkupationen fest, die sich auf das Besondere der schriftstellerischen Praxis von Grass konzentrieren, wobei repräsentative Aspekte wie ,die nichtrealistische Einbildungskraft ${ }^{46}$ und ,barocke Bildfülle ${ }^{47}$ - auch im Umgang mit der Wirklichkeitsbearbeitung ${ }^{48}$-, der nicht wegzudenkende Bestandteil des Mythos („la part irréductible du mythe“49), „die sinnliche Erzählweise, die Sprachanstrengungen, die Variationen komischer Erzählperspektiven“50 den Ausschlag geben. Letztendlich komme die übliche Aufnahme von Mentions zur Sprache. Erwähnt werden einerseits dem französischen Publikum bekanntes

\footnotetext{
44 Ortuño Stühring (2013) 268.

45 George (2008) 304.

46 Serrier (2006) 84-85.

47 George (2008) 309.

48 Serrier (2006) 84-85.

49 Serrier (2006 ) 84-85.

$50 \quad$ George (2008) 309.
} 
und als deutsch identifizierbares Kulturgut (deutsche Schriftstellernamen, der Barock und der Bildungsroman, usw.), und andererseits Namen berühmter französischer Schriftsteller oder Denker (Rabelais, Camus, Balzac, Proust, Flaubert und Céline, sowie Sartre und Montaigne), „wobei Grass selber viele französische Autoren als Vorbilder und Orientierungspunkte zitiert", wie George zurecht bemerkt. ${ }^{51}$ Die Strategie fördert, wie gesagt, die Aneignung des Fremden in Richtung auf das eigene Publikum und ermöglicht eine mentale Zuordnung des zu rezipierenden Werkes. Im Falle einer „eher selbstreferentielle[n] französische[n] Öffentlichkeit“ mit einem starken Kulturverständnis sei diese Lektüre umso wichtiger. ${ }^{52}$

Dass die inländische oder außerdeutsche Grass-Forschung sich mit der französischsprachigen Blechtrommel-Rezeption schwergetan hat, kann also nicht behauptet werden. Auffallend ist, dass keine dieser Untersuchungen auf die anderen verweist. Schon allein wegen der Synthese der bestehenden Forschungsliteratur scheint eine solche Denkübung wünschenswert. Möglicherweise hat das spätere Interesse an einer grenzübergreifenden Rezeptionsforschung mit der werkimmanenten Globalisierung des Grass'schen Werkes (Tansania, Indien, ... ), die laut den Kritikern erst in den 1980er Jahren einsetzt, zugenommen. Dabei wird eine neuere transnationale bzw. supranationale Haltung der Literaturwissenschaft einen komparatistischen Blick auf die Werkrezeptionsforschung sicherlich gefördert haben.

\section{Le Tambour als Schelmenroman. Ein Querschnitt durch die französische Unterrichtsrezeption}

Indem wir über ein großes Interesse für Grass' Werk in Frankreich sprechen, drängt sich die Frage auf, über welche Blechtrommel wir denn reden. Die Rezeption der Blechtrommel setzt 1961 ein, als die erste Übersetzung von Jean Amsler unter dem Titel Le Tambour ${ }^{53}$ beim renommierten Pariser Verlag Seuil erscheint. Der Übersetzer ist kein Anfänger; er ist es aber nicht, der Grass die Türen des Pariser Verlags öffnet. 1958 wurde Grass der Preis der Gruppe 47 verliehen, was laut seiner Aussage das Interesse des Seuil-Verlags weckte. ${ }^{54}$ Es ist der damalige Pariser Freund Paul Celan, der als Vermittler auftritt, Grass mit

\footnotetext{
$5^{1} \quad$ George (2008) 308 .

$5^{2} \quad$ George $(2008) 304$, vgl. 310.

53 Und nicht wortwörtlich Le tambour de tôle, wie der Schriftsteller Michel Tournier in seiner 1975 erschienenen Besprechung für Le Monde zurecht bemerkte. Tournier (1975).

54 Grass (1979).
} 
Rabelais vertraut macht und ihn mit dem Verlag in Verbindung setzt. ${ }^{55}$ Das Buch erfährt im Laufe der Jahre eine Riesenauflage, wird vielmals gedruckt und zudem als Taschenbuchausgabe herausgegeben. 2009 wird der Roman in einer neuen Übersetzung von Claude Porcell veröffentlicht.

Als wesentliche Vermittlung für das Nachleben von Le Tambour im französischsprachigen Raum gilt die berühmte Verfilmung von Volker Schlöndorff aus dem Jahre 1979. Der Film führte zu einer mit der französischen Übersetzung vergleichbaren Erfolgsgeschichte in Cannes, wo das internationale Filmfestival den Film auszeichnete mit der Palme d'Or und dem Oscar du meilleur film étranger. „Oskar [...] connaîtra une deuxième gloire internationale quand Volker Schlöndorff l'adaptera en 1979 au cinéma [...]“..56 Das Revival für den Roman ließ nicht auf sich warten: Abgesehen von zunehmenden Verkaufszahlen diente das Filmplakat auch als Cover für die französische Ausgabe bei Seuil-Points.

Es würde den Rahmen dieses Beitrags sprengen, auszurechnen, wie viel Prozent der gesamten an deutscher Literatur orientierten Buchbesprechungen in Frankreich Grass und sein Werk einnimmt, aber viel ist das auf jeden Fall ${ }^{57}$. Mehr Bedeutung als der Quantität und dem Messen der chiffrierten Aufmerksamkeit, die Grass und sein berühmter Roman über die Jahre hinweg in der französischen Presse genossen haben, kommt hier dem Rezeptionsmodus in Frankreich zu. Im Folgenden wird das Bild deshalb begrenzt auf ein besonderes Segment der Rezeption in Frankreich, das Unterricht und Ausbildung betrifft, nämlich die Aufnahme 2004 von Le Tambour (in französischer Übersetzung) in das Prüfungsprogramm des Staatsexamens Vergleichende Literatur für zukünftige Lehrer und Dozenten in Frankreich (Agrégation de lettres modernes: littérature comparée). In welcher Gestalt gelangt durch die Agrégation, d.h. durch die Unterrichtsrezeption die Remembrance von Le Tambour in die jüngeren Generationen? Und was sagt diese Aufnahme in den Bildungskanon über die Art und Weise aus, wie dieses wichtige Buch aus der europäischen

\footnotetext{
55 Mannoni (2000) 102.

56 Devarrieux/Lindon (1999).

57 Eine Recherche in der Datenbank Europresse verzeichnet als französischsprachige Quellen (nationale und regionale Presse Frankreichs einbegriffen) im April 2015 eine Anzahl von 1115 Beiträge zu Günter Grass. Berechnet man, dass in 249 davon über Le Tambour gesprochen wird, so könnte man feststellen, dass Grass' Magnum Opus in der gesamten“ französischsprachigen Grass-Rezeption 22,3\% der Kommentare einnimmt. Diese Zahl muss aber sofort relativiert werden, und ist insofern nur relevant, als dass die verfügbaren Quellen untereinander manchmal redundant sind und ab und zu nichts zur Sache tun, d.h. nicht als literaturkritischer Kommentar verstanden werden können.
} 
Literaturgeschichte repräsentiert, imaginiert, nacherzählt und in Erinnerung gerufen wird?

Durch die Agrégation trifft man zu einem bestimmten Zeitpunkt vermutlich die größte gemeinsame Lesergruppe des Romans im Unterrichtswesen an. ${ }^{58}$ Außerdem kann noch ein zur weiteren Gedächtnisbildung des RomanImages erheblich beitragendes Potential betrachtet werden, das die zukünftige Berufserfahrung der Diplomierten (agrégés) in sich birgt. Auch wenn hier Leserreaktionen bzw. der Umgang - synchron oder diachron - der Zielleserschaft mit dem Buch aus Mangel an Daten und Messinstrumenten nicht empirisch erforscht werden können und daher außer Betracht bleiben, kann dennoch interessanterweise nach der programmatischen Steuerung der Leseart, die einen Teil der skizzierten Prüfungsvorbereitung und -situation ausmacht, gefragt werden und ihre Vermittlung untersucht werden. Tatsache ist, dass Le Tambour als Pflichtlektüre unter dem Thema „Echos picaresques dans le roman du xxe siècle" (Pikareske Resonanz im Roman des 20. Jahrhunderts) angeboten wurde und eigentlich in diese Kategorie neben zwei anderen Romanen von internationaler Signifikanz - Louis-Ferdinand Célines Voyage au bout de la nuit und Ralph Elissons Invisible Man - eingeordnet wurde.

Allein die Tatsache, dass Le Tambour als repräsentativ für die Frage des Pikaresken in der Literatur des 20. Jahrhunderts gehalten wurde und in das Agregationsprogramm aufgenommen wurde, ist vielsagend für die Sichtbarkeit des Buches mehr als 40 Jahre nach seiner Publikation. Es bezeugt - übrigens nicht nur für Die Blechtrommel, sondern, wie bereits gesagt, schon eher für Ein weites Feld - die Lebhaftigkeit und den Erfolg des kulturellen Transfers um Grass' Werk in Frankreich und stellt in dieser Hinsicht eine Form der transnationalen Kanonisierung dar, und zwar wie eine bestimmte Kollektivität sich dazu entscheidet, ein kulturelles Produkt in Erinnerung zu rufen und in ein (im)materielles Gedächtniserbe aufzunehmen. So betrachtet, funktionieren die Agrégation und ihre Beratungsorgane als Erinnerungsort im Sinne von Pierre Nora, nämlich als Platzhalter für das kollektive Gedächtnis: Jurymitglieder und Teilnehmer sind verantwortlich für das Organisieren, das Übermitteln und das dynamische Verarbeiten einer in diesem Fall offiziellen Form des Gedächtnisses, die hier über die eigene literarische Identität hinaus ein bestimmtes Bild des fremden kulturellen Produkts mitkonstruiert.

$5^{8}$ In welchen unteren Ausbildungsstufen würde dieser schwierige und umfangreiche Roman dem Fremdsprachenunterricht Deutsch bzw. Literaturstudierenden sonst auf nationaler Ebene vorgelegt? 
$\mathrm{Zu}$ fragen ist denn auch, welche Resemantisierung von Le Tambour im französischen Sprachraum unter Berücksichtigung des vorgelegten pikaresken Vergleichsmusters stattgefunden hat. Wie schließen sich dieses Stadium und diese Form der Unterrichtsrezeption an die journalistische bzw. literaturkritische Vorgeschichte an? Inwiefern trägt diese offizielle, kollektiv nach außen getragene und das Pikareske betonende, Herangehensweise als Form der kollektiven Überlieferung an die nächsten Generationen Spuren des historischen literaturkritischen Rezeptionsprozesses? Denn wäre in der programmatischen Profilierung von Le Tambour als Schelmenroman eine Verlängerung bzw. Verabschiedung der ästhetisch orientierten frühen Buchrezeption zu sehen, wäre hierin vielleicht nachträglich ein Grund abzulesen, weshalb der Roman positiv bewertet wurde und damals einen besonderen und unmittelbaren Anschluss an Frankreichs literarisches Feld gefunden zu haben scheint. Indem Le Tambour den Effekt einer Offenbarung hatte, geschah dies laut eines Kritikers eben wegen „le destin picaresque du petit garçon qui refuse de grandir $[. .$.$] “. { }^{59}$ Oder soll man eher von einer Verschiebung und dem Setzen von neuen Akzenten reden? Welche Schelmenaspekte der europäischen Tradition werdenhieraufgegriffen, undwelcheliteraturhistorischen Orientierungspunkte wurden besonders in Bezug auf die französische bzw. deutsche Literatur in den Vordergrund gerückt oder eventuell in den Hintergrund gedrängt? Und was sagt eine solche Darstellung eventuell über den Sinn dieser pikaresken Identität für die französische literarische Institution, ihren punktuellen Umgang mit dem Buch und ihre eigene Entwicklung aus? Auch wenn diese Fragen nicht alle im Nachhinein beantwortet werden können, so sei hiermit das Ausmaß der Problematik der Rezeption literarischer Werke über den Bildungskanon angedeutet.

Wenn das lebendige Gedächtnis durch das Prisma der Leser hier außer Betracht bleibt, sei dennoch auf nachvollziehbare Gedächtnisformen hingewiesen, die im vorliegenden Fall über schriftliche Quellen ermittelt wurden und in diesem Sinne zu erforschen sind. Als Untersuchungskorpus dient hier die schon erwähnte als mögliche Examensvorbereitung angelegte Begleitmonographie. ${ }^{60}$ Als Zeugnisdokument verzeichnet das Buch eine bestimmte eingehende literaturkritische Auseinandersetzung mit Grass' Roman, die in einer Gemeinschaft vorhanden ist, wohl aber keine allgemeine Gültigkeit

59 Guissard (2000).

6o Pinçonnat Crystel, Serrier Thomas, Tettamanzi Régis, Echos picaresques dans le roman du xxème siècle. Editions Atlande, 2003. Siehe zu Grass und seinem Roman als generelle Einführung S. 61-74 und zu einer ausführlichen Analyse des Pikaresken in Grass' Roman vor allem S. 147-168. Die Teile über Grass sind von Thomas Serrier verfasst. 
besitzt: Es bietet einen singulären reflektierten Zugang zum pikaresken Lektüremodus, wozu Leser eingeladen bzw. aufgefordert werden und den die Auszubildenden sich zu eigen machen sollten. In diesem Sinne können kaum Behauptungen über den Kontext, in dem diese Quelle benutzt wird, ihre Dauer, ihr Publikum, ihre Auflage und Verbreitung bei der Vorbereitung des Examens usw. aufgestellt werden. Als verfügbare Quelle von bedeutendem Umfang und thematischer Signifikanz kann sie nicht als repräsentativ für die Art und Weise, wie sich eine bestimmte fremdsprachige Gemeinschaft Le Tambour vorstellt, gehalten werden ${ }^{61}$ Sie wird hier nicht herangezogen, um sich auszusprechen über die Richtigkeit der vorangetriebenen pikaresken Lektüre oder um diese in ihrer Gültigkeit zu positionieren, sondern wird als historisches Rezeptionsmaterial betrachtet, das interessanterweise über die Grenzen der üblichen journalistischen bzw. akademisch-wissenschaftlichen literaturkritischen Kanäle hinausgeht. Ziel ist es also hier, diese Form der Rezeption kritisch zu signalisieren und darüber zu reflektieren, wie sich Formen der interkulturellen Mediation differenziert über die Jahre hinweg und über Generationen entfalten können.

Vor diesem Hintergrund ist insbesondere danach zu fragen, welchen Bezug das pikareske Rezeptionsmuster mitsamt der Begleitpublikation im Kontext der Agregation zur journalistischen Rezeption hat. Erstaunlicherweise kann nicht behauptet werden, dass der pikareske Lektüremodus eine häufige oder sehr gängige Leseart in der Rezeption darstellt; zumindest wenn die Ergebnisse des Suchauftrags zu Grass und Le Tambour in quantitativer Hinsicht für repräsentativ gehalten werden können und wenn alle relevanten Quellen exzerpiert worden sind. In nur 18 von über 249 Quellen aus der nationalen und regionalen Presse Frankreichs, in denen von Le Tambour die Rede ist, kommt ein pikareskes Merkmal des Romans zum Ausdruck. In den 18 untersuchten Quellen kann man die Konturen eines pikaresken Rezeptionsansatzes skizzieren.

Le Tambour wird als Schelmenroman vorwiegend durch die Bestimmung einer ästhetischen und literarischen Abstammung gelesen, die nachdrücklich auf die spanischen Pikaresken und die deutsche Barockzeit (Grimmelshausen) Bezug nimmt, worauf noch der entscheidende Schatten von Rabelais fällt. ${ }^{62}$ Grass selber habe in Interviews, auch anlässlich späterer Publikationen wie Ein weites Feld, mehrmals auf die Wichtigkeit der europäischen Tradition des Schelmenromans als ästhetische Vorlage seines Werks hingewiesen. Bemerkenswert ist, dass er dabei immer wieder als ,'lauteur du Tambour' präsentiert

\footnotetext{
61 Sie entstammt auch vorwiegend, wie gesagt, dem Denken eines einzigen Literaturwissenschaftlers und -Vermittlers, Thomas Serrier.

62 Simon (1961); Schneider/Corty (1999); Deshusses (1999).
} 
wird. ${ }^{63}$ Auch Le Turbot (Der Butt, 1977), sein vielleicht zweitberühmtestes Buch nach Die Blechtrommel, wird als Rückkehr von Grass zu einer ,littérature picaresque bezeichnet. ${ }^{64}$

Bei der Blechtrommel erinnert die Umkehr der Perspektive des Bildungsromans mittels der winzigen Gestalt eines Kindes und dessen ,zersingender ${ }^{\star}$ Erzählstimme Tournier eben an den Simplicissimus von Grimmelshausen, ${ }^{65}$ der tatsächlich zu Grass' bekannten und vielzitierten Vorbildern gehört. So ist noch für den einen das Schicksal des kleinen Oskars von pikaresken Zügen geprägt, ${ }^{66}$ während für den anderen die Froschperspektive, die die Geschichte von unten und vom Standpunkt der Verlierer aus darstellt, „dem Stil des Schelmenromans verpflichtet" ist. ${ }^{67}$ Auffallend ist schließlich die Ausbreitung des Pikaresken als Rezeptionsmuster der Erzählung über die Verfilmung Schlöndorffs: „Comme le roman de Günter Grass, le film de Schlöndorff tient [...] du récit picaresque“.68 Bis in Fernsehprogramme und Tv-Filmankündigungen in wichtigen nationalen Zeitungen wie Le Monde wird Jahr für Jahr (1992, 1996, 1998, 1999, 2011) wortwörtlich von einem ,grand film picaresque gesprochen.

Bemerkenswert ist noch, dass Grass an anderen Stellen als derjenige genannt wird, der selbst seit Le Tambour zur Nachwirkung der Schelmentradition beigetragen habe. So wurden zeitgenössische französische Schriftsteller (wie René-Victor Pilhes z.B.) und ihre damalige Romanproduktion bereits 1965 und später nochmals 1975 am Maßstab des Pikaresken, mit dem Grass' Werk assoziiert wird - „la grande tradition mouvementée, captivante, du picaresque“, gemessen. ${ }^{69}$

Zum Schluss stellt sich noch die im Kontext dieser Publikation interessante Frage, ob die spätere Aufnahme des Romans in einen pikaresken Bildungskanon der europäischen Literaturgeschichte Geistesverwandtschaft aufzeigt mit der skizzierten journalistischen Rezeption. Bemerkenswert ist, dass die Relevanz des ,aufgelegten' pikaresken Themas von vornherein in Frage gestellt und dieses nicht als selbstverständlich diskutiert wurde: zum einen aufgrund der laut mancher Kritiker (Mannoni) problematischen Annäherung an Céline (Serrier), mit dem Grass oft verglichen wurde, von dem er sich selber aber in Interviews mehrmals ausdrücklich distanziert hat und über den Jean-Pierre Lefebvre in

\footnotetext{
63 u. a. Cory (1997).

64 Devarrieux/Lindon (1999).

65 Tournier (1975).

66 Guissard (2000).

67 Vernet (2005) [Übersetzung von S. V.].

68 De Baroncelli (1979); s. auch Siclier (1999).

69 Piatier (1965) u. Tournier (1975).
} 
seinem Vorwort zur Pocketausgabe von Le Tambour (1999, ,Points') schrieb, dass sein ,envol lyrique' Grass' Werk fremd sei; zum anderen wegen des Exports der historischen Kategorie des Pikaresken, bis weit in das 20. Jahrhundert hinein..$^{70}$ Der pikareske Weg, den die wissenschaftliche Publikation folglich in Le Tambour und andeutungsweise in späteren Grass'schen Werken wie Der Butt oder Ein weites Feld bewandert, lässt das journalistische Rezeptionsmuster eher unbeachtet, und knüpft auch nicht an historisch vorhandenen literaturkritischen Kommentaren an. Die von Serrier präsentierte Analyse erarbeitet eine davon unabhängige Reflexion zu den Spuren der Schelmenperspektive in Grass' Roman.

Ohne hier auf die Einzelheiten dieser pikaresken Deutung einzugehen, kann man, allgemein betrachtet, mit Serrier hinsichtlich einer Rückkehr der Schelmenliteratur im 20. Jahrhundert festhalten, dass Grass mit dem Erscheinen der Blechtrommel in Deutschland einen doppelten poetologischen Paradigmenwechsel verursacht hat: zum einen bezüglich der herrschenden Trümmerliteratur der Nachkriegszeit, zum anderen, weil er den großen deutschen Klassikern und Erzählern ihren epischen Atem entlieh, um sie besser auf den Kopf zu stellen. Dagegen herrschte damals in Paris, woran auch Thomas Serrier erinnert, ${ }^{71}$ die Mode des Nouveau Romans, der die Zersplitterung der Erzählung und die Desakralisierung der tradierten Romanformen vorantrieb. So betrachtet hat die von Grass im literarischen Deutschland verursachte Zäsur in Frankreich vielleicht eher einen Eindruck des schon Dagewesenen hinterlassen. Die Anhäufung von zerbröckelten Erzählstoffen, die ineinander geschoben werden und als Sequenzen montiert werden, ist nämlich der pikaresken Gattung nicht fremd. Folgende Aussage Serriers könnte denn als symptomatisch gelten: „Ce que l'on entend par picaresque dans Voyage au bout de la nuit, Invisible Man et Le Tambour relève d'une perception générique, d'une impression de déjà vu. “72 Zu diesem Déjà-vu trugen auch in Grass' Roman selbstverständlich noch Spuren des Humors und der Farce, die für das französische Publikum schon in der mittelalterlichen Tradition von Till Eulenspiegel und seinen pfiffigen Figuren verkörpert war, ${ }^{73}$ sowie seine an Rabelais erinnernde Wortfülle und seine skatologischen Exzesse, bei.

So zeigt der pikareske Lektürevorschlag, der nicht zufällig der Verleihung des Nobelpreises folgte, die Grass als europäischen Autor zelebrierte, wie der Roman nicht konkret thematisch als Verlängerung des französisch-

\footnotetext{
$70 \quad$ Serrier in Pinçonnat e.a. (2003) 11.

71 Serrier in Pinçonnat e.a. (2003) 154.

72 Serrier in Pinçonnat e.a. (2003) 27.

73 Serrier in Pinçonnat e.a. (2003) 150.
} 
sprachigen literaturkritischen Rezeptionsansatzes gelesen wurde, sondern eher die allgemeine Tendenz zur ästhetisierenden Aufnahme, die schon die frühe journalistische Rezeption kennzeichnete, vertiefte. Somit wurde Le Tambour weniger in Bezug auf landeskundliche, historische oder nationalistische Aspekte rezipiert, sondern eher in einer ästhetischen Perspektive weitersemantisiert, und zwar als Schelmenroman innerhalb einer europäischen Tradition, in der eigene Modelle wie - neben Céline - Rabelais oder Till l'Espiègle - weiterleben.

\section{Literatur}

Anon., „La sélection de la semaine:Jeudi 29 octobre, ,Le Tambour“', in Le Monde, ctober $26,1992,5$.

Anon., „Le Monde Télévision: Le Tambour“, in Le Monde, January 28, 2011.

Ballestra-Puech, S., ,L'art des titres ou le mélange des genres dans Le Tambour de Günter Grass", in Loxias 7, December 15, 2004, <http://revel.unice.fr/loxias/index.html? id $=90>$.

Bjurstrom, C., „Des romanciers réalistes et véridiques ... “, in Le Monde, April 07, 1975.

Braun, R./F. Brunssen, Changing the Nation. Günter Grass in international Perspective (Würzburg, 2008).

Casanova, N., „J'ai tiré parce que je suis allemand ... “, in: La Quinzaine littéraire, nr. 841, December 1, 2002.

Corty, B., „La vie littéraire. La revue des revues [über L'atelier du roman, N 12, 85 F. rendant hommage à Günter Grass]", in Le Figaro littéraire, October 23, 1997, 2.

De Baroncelli, J., „Le jour où Oskar cessa de grandir“, in Le Monde, September 22, 1979.

Deshusses, P., „L'unité mis à plat. Envoûtant, agaçant, le roman sans doute le plus difficile, et le plus allemand de Günter Grass, mais aussi le plus universel et le plus généreux", in Le Monde des Livres, October 03, 1997.

Deshusses, P./G. Grass, „Günter Grass, en dissonance“, in Le Monde. Le Monde des livres, October 03, 1997, 1 .

Deshusses, P., „Prix Nobel de littérature attribué le jeudi 30 septembre 1999. Günter Grass, écrivain des dissonances de l'histoire allemande“, in Le Monde, October 02, 1999, 30.

Deshusses, P., „Günter Grass. Ecrivain allemand, Prix Nobel“, in Le Monde, April 15, 2015, 16.

Devarrieux, C./M. Lindon, „L'an de Grass. Le dernier Nobel du siècle couronne le plus radical des écrivains allemands", in Libération, October 01, 1999, xiii.

Espagne, M., „La notion de transfert culturel“, in Revue Sciences/Lettres [online], 1 | 2013, URL : <http://rsl.revues.org/219; DOI : 10.400o/rsl.219>. [Zugriff: Januar 2016]. 
Fritzen W., „Besprechung von Harro Zimmermann, Günter Grass unter den Deutschen“, in Germanistik 47/3-4, 2006, 96.

George, M., „Günter Grass und Frankreich“, in Honsza, N./I. Swiatlowska (Hg.), Günter Grass. Bürger und Schriftsteller (Wroclaw, 2008), 297-312.

Grass, G., „Rückblick auf die Blechtrommel - oder Der Autor als fragwürdiger Zeuge. Ein Versuch in eigener Sache“, in: Grass, G., Essays und Reden II (Göttingen, 1997), $323-332$.

Grass, G., „Im Ausland geschätzt, im Inland gehasst“, in Grass, G., Der Schriftsteller als Zeitgenosse (Göttingen, 1997).

Grass, G., Danziger Trilogie / Die Blechtrommel (Darmstadt/Neuwied, 1980), 287.

Grass, G, L'atelier des métamorphoses. Entretiens avec Nicole Casanova (Paris, 1979).

Grass, G./H. Zimmermann, Vom Abenteuer der Aufklärung. Werkstattgespräche (Göttingen, 1999).

Grass, G., Le Tambour (Paris, 2013) (1961 erste Ausgabe der französischen Übersetzung, 1997 für die Buchpräsentation von Jean-Pierre Lefebvre).

Guissard, L., Itinéraire d'un anticonformiste. Une biographie remarquable de Günter Grass, écrivian allemand engagé [über Günter Grass. L'honneur d'un homme von Olivier Mannoni], in La Croix, September 28, 2000, 16.

Joosten, J., Staande receptie (Nijmegen, 2012).

Lartillot, F., Günter Grass' Ein weites Feld. Aspects politiques, historiques et littéraires. Actes du colloque de Nancy. Centre de Recherche Germaniques et Scandinaves de l'Université de Nancy II. Bibliothèque Le Texte et l'Idée, vol. X, 2001.

Mannoni, O. Günter Grass, L'honneur d'un homme (Paris, 200o).

Mews, S., Günter Grass and His Critics: From The Tin Drum to Crabwalk, 2008.

Ortuño Stühring, C., Die Kritik der Gegenwart. Eine systematische Analyse deutsch-französischer Literaturkritik am Beispiel von Michel Houellebecq und Günter Grass (München, 2013).

PIatier, J., „La Rhubarbe', de René-Victor Pilhes“, in Le Monde, September 11, 1965.

Pinçonnat C./T. Serrier/R. Tettamanzi, Echos picaresques dans le roman du xxème siècle. Editions Atlande, 2003.

Quéval, M. (Hg.), Lectures d'une œuvre : Ein weites Feld. Günter Grass (Paris, 2001).

Schneider, M./B. Corty, „L'auteur du ,Tambour' obtient le 96e prix Nobel de littérature. Günter Grass, l'enfant terrible des lettres allemandes“, in Le Figaro, October 01, 1999, 36.

Schwarz, W. , Der Erzähler Günter Grass (Bern, 1969).

Serrier, T., „Günter Grass et la France“, in Atala 9, 2006.

Siclier, J., „Le Monde télévision radio multimédia. La critique de Jacques Siclier“, in Le Monde, March 11, 1996, 22.

Siclier, J., „Le Monde télévision radio multimédia. Les films de la semaine par Jacques Siclier“, in Le Monde, June 01, 1998, 23. 
Siclier, J., „Le Monde télévision radio multimédia. La critique de Jacques Siclier“, in $L e$ Monde, March 11, 1996, 22.

Siclier, J., „Le Monde télévision radio multimédia. Samedi 17 juillet, film de Volker Schlöndorff: Le Tambour“, in Le Monde, July 12, 1999, 28.

Simon, P., „Le Tambour de Günter Grass“, in Le Monde, December 27, 1961.

Tournier, M., „,Le Tambour' relu par ,Le Roi des Aulnes““, in Le Monde, January 17, 1975.

Wellnitz, P., Günter Grass: Ein weites Feld/ Toute une histoire (Strasbourg, 2001). 